\title{
NEUTROPHIL ENDOPEPTIDASE INHIBITOR IMPROVES PULMONARY FUNCTION DURING REPERFUSION AFTER EIGHTEEN-HOUR PRESERVATION
}

Oliver A. R. Binns, MD

Nuno F. Delima, MD

Scott A. Buchanan, MD

Michael C. Mauney, MD

Jeff T. Cope, MD

Stephen D. Thies, MD

Kimberly S. Shockey, MS

Curtis G. Tribble, MD, FACS

Irving L. Kron, MD, FACS
Background: Reperfusion injury remains a significant problem after lung transplantation and is thought to be in part mediated by neutrophils. Ulinastatin inhibits release of elastase and cathepsin $G$ from neutrophil granules. We hypothesized that inhibition of these neutrophil endopeptidases (proteases) would attenuate pulmonary reperfusion injury. Methods: With an isolated, whole blood-perfused, ventilated rabbit lung model, we studied the effects of ulinastatin. All lungs were flushed with cold EuroCollins solution, harvested en bloc, stored inflated at $4^{\circ} \mathrm{C}$ for 18 hours, and reperfused with whole blood. The 18-hour control lungs $(n=8)$ were stored and reperfused. Low-dose $(n=8)$ and high-dose $(n=7)$ groups were treated with total doses of ulinastatin of 25,000 and 50,000 units, respectively, during flush and reperfusion. An additional control group of lungs $(n=8)$ was harvested, flushed, and immediately reperfused. Results: The pulmonary artery pressure was significantly lower in the high-dose group than in the 18-hour control group $(36.7 \pm 1.8$ vs $44.8 \pm 2.9 \mathrm{~mm} \mathrm{Hg}, p=$ $0.034)$. The percentage decrease in dynamic airway compliance was significantly less in the high-dose group than in the 18-hour control group $(-13.8 \% \pm 4.4 \%$ vs $-25.1 \% \pm 3.7 \%, p=0.032)$. Both low-dose and high-dose ulinastatin treatments did not result in a significant improvement in oxygenation with respect to the 18-hour control group $(72.2 \pm 25.8$ vs $32.5 \pm 4.9 \mathrm{~mm} \mathrm{Hg}, p=0.21$ ). Conclusions: Ulinastatin diminishes reperfusion injury after 18 hours of hypothermic pulmonary ischemia, with resultant improvements in pulmonary artery pressure and airway compliance. Improvement in pulmonary function after preservation and reperfusion with a neutrophil endopeptidase inhibitor confirms the role of endopeptidases in reperfusion injury and suggests an intervention to reduce their detrimental effects on early graft function. (J Thorac Cardiovasc Surg 1996;112:607-13)
From the Division of Thoracic and Cardiovascular Surgery, Department of Surgery, University of Virginia Health Sciences Center, Charlottesville, Va.

Supported by the National Institutes of Health under RO1 grant HL 48242 and NRSA fellowship F32HL09115-01A1. Additional support from $\mathrm{CNPq}$ - Conselho Nacional de Desenvolvimento Cientifico e Tecnologico, Brazil.

Presented in part at the Surgical Forum, American College of Surgeons, Oct. 25, 1995.

Received for publication Nov. 1, 1995; revisions requested Jan. 4, 1996; revisions received Jan. 24, 1996; accepted for publication Jan. 4, 1996

Address for reprints: Irving L. Kron, MD, Division of Thoracic and Cardiovascular Surgery, Department of Surgery, Box 310, University of Virginia Health Sciences Center, Charlottesville, VA 22908 .

Copyright (C) 1996 by Mosby-Year Book, Inc.

$0022-5223 / 96 \$ 5.00+0 \quad \mathbf{1 2 / 1 / 7 2 5 6 5}$
Deperfusion injury remains an important cause of Rimpaired lung function after transplantation. This injury is believed to be partially mediated by the deleterious effects of neutrophils. The use of leukocyte-depleted blood during heart-lung transplantation has resulted in improvement in functional and histologic indexes. Successful lung transplantation after extended preservation with leukocyte-depleted blood has also been described. ${ }^{2}$ The concept of host responses contributing to pathologic processes is widely accepted. Tissue destruction by neutrophils has been attributed to their ability to release "a complex assortment of agents that can destroy normal cells and dissolve connective tissues." 3 This process is initiated by pulmonary endothelial injury as a consequence of ischemia, poor preservation, and reperfusion of the lung. 
During reperfusion, endothelial cells express intercellular adhesion molecules and endothelial leukocyte adhesion molecule, facilitating neutrophil adherence, activation, and subsequent toxin release. ${ }^{4}$ These neutrophil-generated toxins can be divided into two categories: reactive oxygen metabolites generated from the plasma membrane through the reduced nicotinamide adenine dinucleotide oxidase system and preformed endopeptidases (proteases) released from intracellular granules.

Numerous investigations have demonstrated the role of reactive oxygen metabolites in reperfusion injury of pulmonary tissues after preservation ${ }^{5,6}$; however, the role of neutrophil endopeptidases in reperfusion injury of the lung has yet to be elucidated. The aim of our study was to investigate the effect of these neutrophil endopeptidases on lung function after preservation and reperfusion. To accomplish this, we used the neutrophil endopeptidase inhibitor ulinastatin (gift of Mochida Pharmaceutical Co., LTD., Tokyo, Japan), which inhibits the actions of elastase and cathepsin $\mathrm{G}$ released from neutrophil granules. We hypothesized that inhibition of these neutrophil endopeptidases would attenuate reperfusion injury and thus improve pulmonary function after 18 hours of cold ischemia.

\section{Materials and methods}

Lung-heart block harvesting. With the following experimental model previously described elsewhere, ${ }^{7} 31$ adult New Zealand white rabbits of both sexes $(3.0$ to $3.5 \mathrm{~kg}$ ) were randomly assigned to four experimental groups. Each rabbit was anesthetized with intramuscular ketamine $(50 \mathrm{mg} / \mathrm{kg})$ and xylazine $(5 \mathrm{mg} / \mathrm{kg})$. Tracheal intubation was performed through a tracheostomy and followed by paralysis with intravenous metocurine $(0.2 \mathrm{mg} / \mathrm{kg}) . \mathrm{Me}-$ chanical ventilation was instituted (Ventilator RSP1002; Kent Scientific Corporation, Litchfield, Conn.) with room air at a tidal volume of $12 \mathrm{ml} / \mathrm{kg}$ and a rate of 20 breaths/min.

A median sternotomy and a thymectomy were then performed. The superior and inferior venae cavae were loosely encircled with ligatures and the pericardium was opened. Both the pulmonary artery (PA) and the aorta were dissected free and similarly encircled. A purse-string suture was then placed in the free wall of the right ventricle and intravenous heparin was administered (500 $\mathrm{U} / \mathrm{kg}$ ). After injection of $30 \mu \mathrm{g}$ prostaglandin $\mathrm{E}_{1}$ (Alprostadil; Upjohn Company, Kalamazoo, Mich.) directly into the $\mathrm{PA}$, the venae cavae were ligated to initiate the 18-hour ischemic period.

The PA was then cannulated through a right ventriculotomy in the center of the purse-string suture, and both the right ventricular and PA ligatures were tied around the cannula. After the left ventricle was vented through a left ventriculotomy and the aorta was ligated, $50 \mathrm{ml} / \mathrm{kg}$
Euro-Collins solution at $4^{\circ} \mathrm{C}$ was infused into the PA from a height of $30 \mathrm{~cm}$. Topical cooling was achieved with cold saline solution slush. During PA flush, the left atrium (LA) was cannulated through the left ventriculotomy and a second purse-string suture was tied around the cannula. A second catheter was placed in the LA to directly transduce LA pressures. In the experimental groups designated to receive ulinastatin, the endopeptidase inhibitor was administered with the final $15 \mathrm{ml}$ flush solution and again at the onset of reperfusion. After the PA flush, the inflow and outflow cannulas were clamped. Care was taken to leave the pleurae intact until completion of the flush, to avoid parenchymal injury. The lungs were stored inflated by clamping of the tracheal tube at end-inspiration. The lung-heart block was then excised, immersed in cold $0.9 \%$ saline solution, and stored at $4^{\circ} \mathrm{C}$ for 18 hours. All experimental protocols were reviewed and approved by an institutional animal use committee. All animals received humane care in compliance with the "Guide for the Care and Use of Laboratory Animals" prepared by the Institute of Laboratory Animal Resources and published by the National Institutes of Health (NIH publication No. 85-23, revised 1985).

Assessment of lung function. After 18 hours of storage at $4^{\circ} \mathrm{C}$, the lung-heart block was suspended by a force transducer in a warm, humidified tissue chamber. Ventilation was reestablished with a $95 \%$ oxygen $5 \%$ carbon dioxide gas mixture at a tidal volume of $12 \mathrm{ml} / \mathrm{kg}$ and a respiratory rate of $20 \mathrm{breaths} / \mathrm{min}$. The lungs were reperfused with homologous fresh whole venous blood from a main reservoir. A second venous blood reservoir was used to determine single-pass oxygenation values at 10,20 , and 30 minutes after initiation of reperfusion. Blood was harvested from a single rabbit for each experiment. The inflow and outflow cannulas were then connected to the blood-primed perfusion circuit, with care taken to avoid introduction of air. The perfusion circuit (Kent Scientific Corporation) was designed to recirculate $150 \mathrm{ml}$ warmed blood through a $270 \mu \mathrm{m}$ blood filter (2C7600; Baxter Healthcare Corp., Deerfield, Ill.) with a roller pump (7521-40; Cole-Parmer Instrument Company, Niles, Ill.) at a rate of $60 \mathrm{ml} / \mathrm{min}$ (Fig. 1). A $270 \mu \mathrm{m}$ blood filter was chosen to avoid affecting leukocyte and platelet counts. Continuous recordings of PA pressure (PAP), LA pressure, lung weight, airway flow, and airway pressure were facilitated by a dynamic data-acquisition program (Workbench PC; Strawberry Tree, Inc., Sunnydale, Calif.) run on a personal computer (470A; Compaq Prolinea, Houston, Texas). This program automatically calculated and displayed pulmonary vascular resistance (PVR), tidal volume, and dynamic airway compliance. LA pressure was maintained within the physiologic range (4 to $8 \mathrm{~mm} \mathrm{Hg}$ ) by adjusting the height of a small outflow reservoir in the circuit. Pulmonary venous blood samples were collected for blood gas analysis (Corning $178 \mathrm{pH} /$ Blood Gas Analyser; Corning Inc., Corning, N.Y.) at 10, 20, and 30 minutes after the start of reperfusion. At each sampling interval, inflow from the main reservoir was interrupted and the circuit was filled with venous blood from the second inflow reservoir. A $30 \mathrm{ml}$ sample of venous blood was passed through the pulmonary vasculature at each interval to ensure accurate measurement of pulmonary 


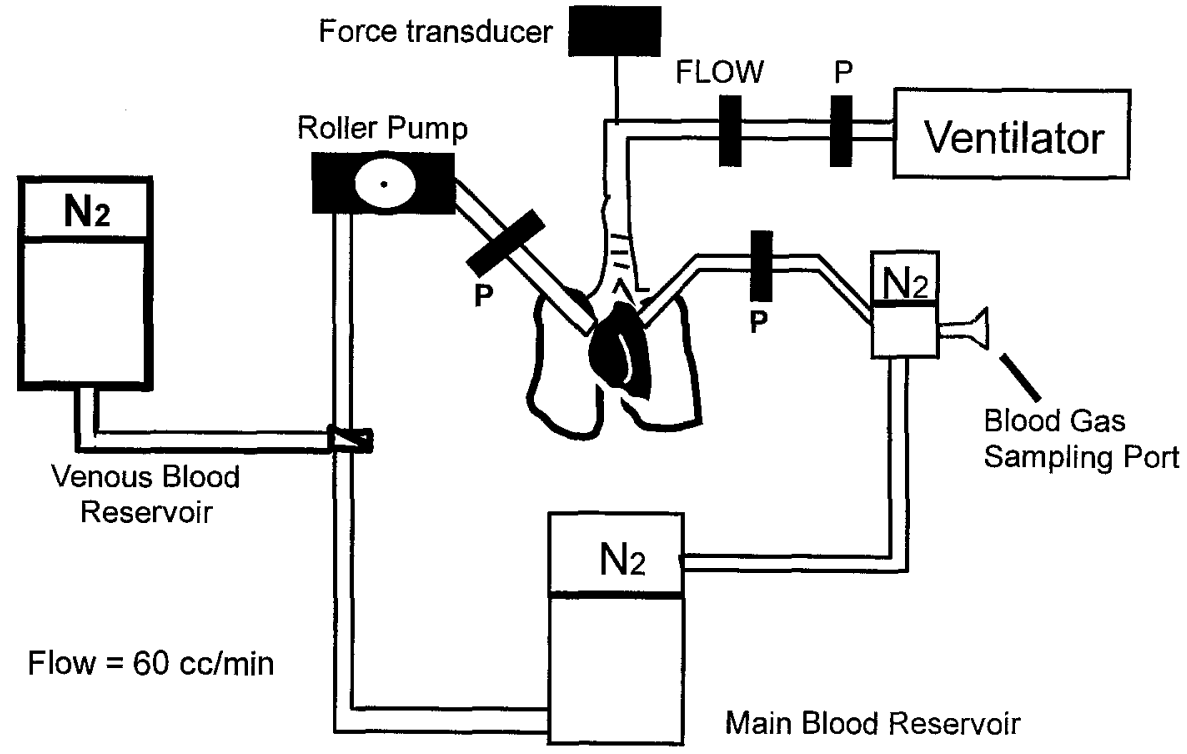

Fig. 1. Schematic diagram of the model. $P$, Pressure transducer.

venous oxygen content. Oxygen contact with exposed blood surfaces inside the reservoir containers was minimized by continuous infusion of $100 \%$ nitrogen. After 30 minutes of reperfusion, specimens of lung tissue were acquired for weight analysis. Wet-to-dry ratios were calculated after passive desiccation at room temperature to a stable dry weight.

Experimental protocol. All lungs were flushed with Euro-Collins solution and reperfused at a physiologic flow rate of $60 \mathrm{ml} / \mathrm{min}$ for 30 minutes. Four experimental groups were defined as follows: 18-hour control group lungs $(n=8)$ were reperfused without intervention after 18 hours of storage, low-dose group lungs $(n=8)$ received 2500 units ulinastatin during flush and 22,500 units at reperfusion, and high-dose group lungs $(n=7)$ received 5000 units ulinastatin during flush and 45,000 units at reperfusion. The dosage of ulinastatin was determined according to the manufacturer's recommendations for its use in clinical human shock states. An additional immediate control group $(n=8)$ underwent reperfusion immediately after flushing. Data were recorded every $15 \mathrm{sec}-$ onds and analyzed at the end of the 30-minute reperfusion period. Oxygenation data were obtained and analyzed at 10 -minute intervals. All values are expressed as means ( \pm standard error of the mean). Statistical analysis was performed by analysis of variance to compare the experimental groups. A difference was considered statistically significant if the $p$ value was less than 0.05 .

\section{Results}

All lungs demonstrated evidence of reperfusion injury when compared with the immediate control group. These changes were manifested as significant increases in PAP, PVR, and wet-to-dry ratios, in addition to significant decreases in airway compli- ance and oxygenation capacity. In our model, we defined oxygenation capacity as the ability of the lungs to oxygenate venous blood during a single pass from the PA to the LA. This was reported as a venous-arterial gradient $\left(\mathrm{V}-\mathrm{AO}_{2}\right)$ to normalize for gradual decreases in the venous blood oxygen tension during the course of the experiment. After 30 minutes of reperfusion, the mean PAP of the highdose group was significantly lower than that of the 18-hour control group $(36.7 \pm 1.8$ vs $44.8 \pm 2.9 \mathrm{~mm}$ $\mathrm{Hg}, p=0.034$ ). There was no significant difference between the PAP of the high-dose group and that of the immediate control group (Fig. 2). Although the mean PVR was lower in the high-dose group, this difference was not significant compared with the 18-hour control group $(43,310 \pm 2044$ vs $51,935 \pm$ 4867 dynes $\left.\cdot \mathrm{sec} \cdot \mathrm{cm}^{-5}, p=0.41\right)$. The high-dose group also exhibited less of a drop in compliance than did the 18-hour control group $(-13.8 \% \pm$ $4.4 \%$ vs $-25.1 \% \pm 3.7 \%, p=0.032$; Fig. 3 ). The 18-hour control lungs demonstrated significantly greater edema formation than did the immediate control group, as determined by higher wet-to-dry ratios (7.44 \pm 0.31 vs $5.50 \pm 0.25, p=0.0014)$. Edema formation, as reflected by wet-to-dry ratios, was not significantly different between the high-dose and 18-hour control groups (6.58 \pm 0.29 vs $7.44 \pm$ $0.31, p=0.35$; Fig. 4). The high-dose group demonstrated a higher mean $\mathrm{V}-\mathrm{AO}_{2}$ gradient than did the 18-hour control group, although this difference 


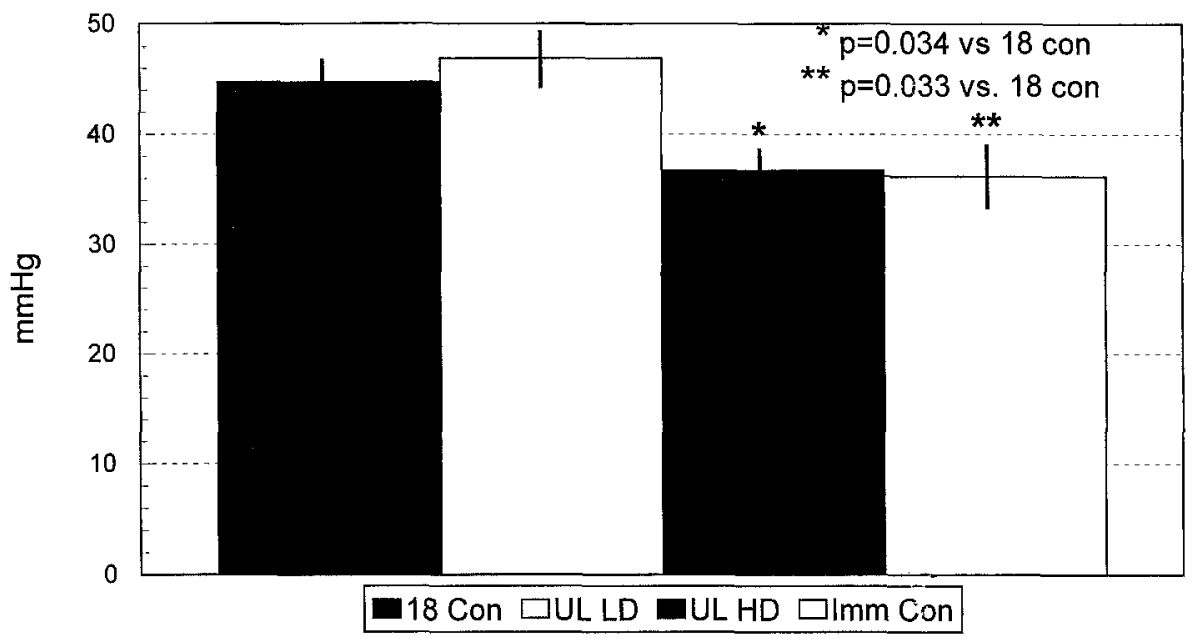

Fig. 2. Differences in PAP after 30 minutes of reperfusion. High-dose ulinastatin group (UL HD) exhibits decreased PAP compared with the 18-hour control group (18 con). UL LD, Low-dose ulinastatin group.

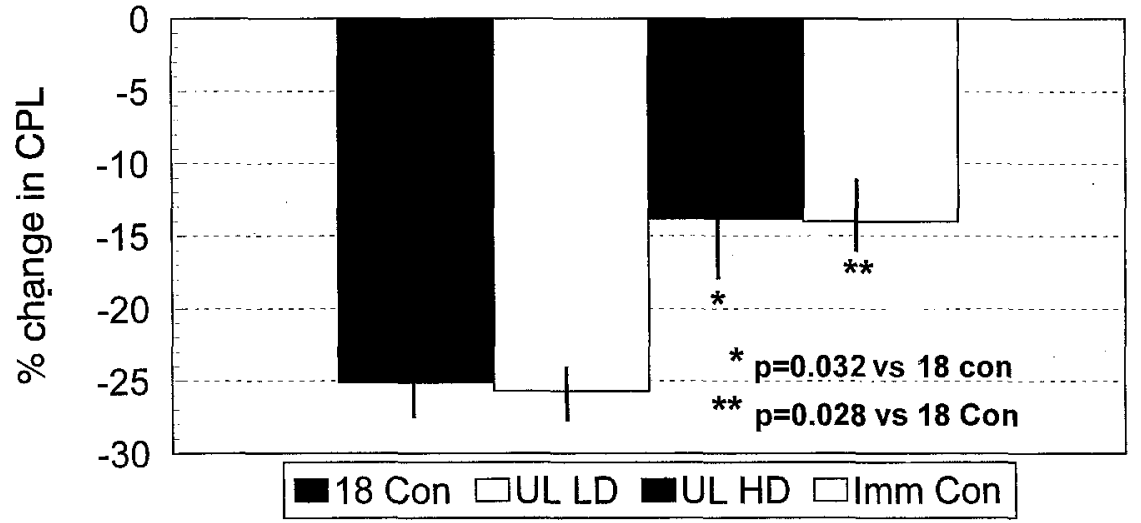

Fig. 3. Changes in lung compliance (CPL) after 30 minutes of reperfusion. High-dose ulinastatin group (UL HD) exhibits less drop in compliance than seen in the 18-hour control group (18 Con). UL LD, Low-dose ulinastatin group; Imm Con, immediate control group.

failed to reach statistical significance $(72.2 \pm 25.8 \mathrm{vs}$ $32.5 \pm 4.9 \mathrm{~mm} \mathrm{Hg}, p=0.21$; Fig. 5). All of the lungs preserved for 18 hours demonstrated markedly decreased oxygenation capacity $\left(\mathrm{V}-\mathrm{AO}_{2}\right.$ gradient) compared with the immediate control lungs, which had a mean $\mathrm{V}-\mathrm{Ao}_{2}$ gradient of $361.3 \pm 32.2 \mathrm{~mm} \mathrm{Hg}$. The low-dose group showed no improvements in PVR, dynamic compliance, wet-to-dry ratios, or $\mathrm{V}-\mathrm{AO}_{2}$ compared with the 18-hour control group ( $\mathrm{V}-\mathrm{AO}_{2}$, $46.9 \pm 6.0$ vs $32.5 \pm 4.9 \mathrm{~mm} \mathrm{Hg}, p=0.62$ ).

\section{Discussion}

Reperfusion injury after lung transplantation continues to contribute to increased morbidity and mortality. Neutrophils are implicated as mediators of this reperfusion injury in the lung and other organs. ${ }^{1-3,8-11}$ Numerous investigators have demonstrated improved pulmonary function after ischemia by employing interventions to modulate neutrophil activity. These include the use of leukocyte-depleted blood ${ }^{8}$ antibodies directed at adhesion molecules expressed on the endothelial surface, ${ }^{12}$ antibodies directed at the leukocyte integrin $\mathrm{CD} 18,{ }^{13}$ agents aimed at preventing the expression of integrin heterodimers on the leukocyte surface, ${ }^{14}$ and oxygenfree radical scavengers. ${ }^{5}$ Morphologic changes seen in lung reperfusion injury are consistent with damage caused by neutrophil endopeptidases ${ }^{15}$ because 


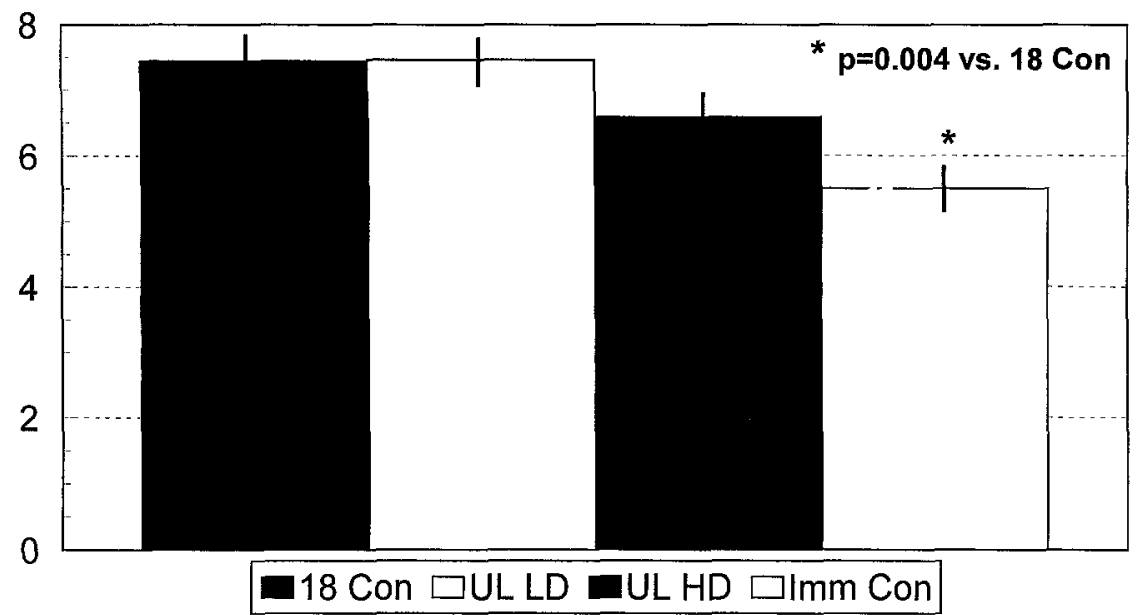

Fig. 4. Wet-to-dry ratios. Evidence of reperfusion injury when comparing 18-hour control group (18 Con) with immediate control group (Imm Con). UL $L D$, Low-dose ulinastatin group; UL HD, high-dose ulinastatin group.

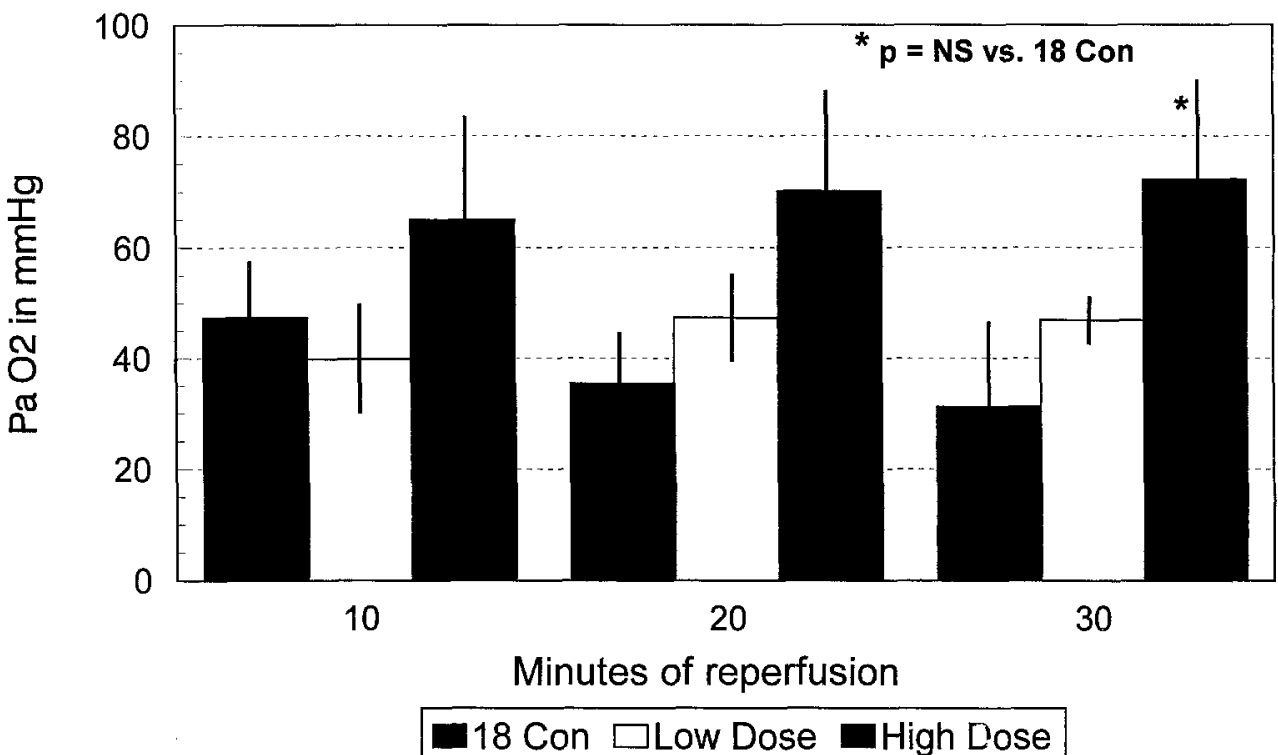

Fig. 5. $\mathrm{V}-\mathrm{AO}_{2} . \mathrm{NS}$, Not significant; $18 \mathrm{Con}$, 18-hour control group; $\mathrm{PaO}_{2}$, arterial oxygen tension.

these proteins are able to destroy the extracellular matrix. Few studies, however, have investigated the specific role of neutrophil endopeptidases in reperfusion injury of the lung. ${ }^{16}$

Under normal physiologic conditions, the action of these preformed enzymes is largely regulated by three antiendopeptidases: $\alpha_{1}$-proteinase inhibitor, $\alpha_{2}$-macroglobulin, and secretory leukoproteinase inhibitor. Evidence exists that chlorinated oxidants generated by neutrophils are able to "destroy the antiproteinase shield allowing unregulated proteolytic enzymes to attack and degrade host tissues." Moreover, it is likely that periods of cold ischemia would attenuate synthesis of these antiendopeptidases in preserved tissues, rendering them more susceptible to the uninhibited action of these endopeptidases.

To investigate the effects of these endopeptidases on pulmonary reperfusion injury, we employed the inhibitor ulinastatin. This glycoprotein isolated from 
human urine has been described as an inhibitory biologic response modifier. ${ }^{17}$ Ulinastatin directly inhibits the actions of elastase and cathepsin G. It also indirectly inhibits the release of neutrophil endopeptidases by stabilizing lysosomal membranes. Ulinastatin has been employed in other organ models of ischemia-reperfusion injury, ${ }^{18-20}$ as well as in previous models of pulmonary injury. ${ }^{6,21}$ Horiguchi and Harada ${ }^{16}$ demonstrated decreased PVR and leukocyte infiltration in their model of pulmonary ischemia and reperfusion by using ulinastatin; however, no measurements of airway dynamics or oxygenation capacity, both of which are important indexes in the study of lung function, were included.

With an isolated, ventilated, perfused ex vivo rabbit lung model, we demonstrated evidence of reperfusion injury in all lungs preserved for 18 hours. This injury was manifested by increases in PAP, PVR, and wet-to-dry ratios, accompanied by decreases in compliance and $\mathrm{V}-\mathrm{AO}_{2}$. Improvements in PAP and lung compliance by treatment with ulinastatin suggest enhanced preservation of the microcirculation's integrity and endothelial function. Despite improvements of these parameters in the high-dose group, a statistically significant improvement in oxygenation with respect to 18 -hour control group was not demonstrated. The ability of lungs to oxygenate the perfusate remains the single most important parameter of lung function. Decreased oxygenation remains a sensitive marker of reperfusion injury, and our results suggest that other mechanisms of injury continue to be involved. The high-dose group did, however, show incremental improvement with time, whereas the 18-hour control group demonstrated decreasing oxygenation as the reperfusion period progressed. This observation suggests ongoing neutrophil endopeptidase activity and worsening injury in the absence of the endopeptidase inhibitor. It also corroborates previous findings of continued leukocyte-mediated injury minutes to hours after the onset of reperfusion.

Similarly, improving oxygenation capacity with time in the low-dose group suggests activity of the endopeptidase inhibitor. The low-dose group failed, however, to show improvements in other parameters of lung function after 18 hours of cold ischemia. This most likely represents an inadequate dosage of ulinastatin in the face of such severe reperfusion injury.

Lung transplantation remains a successful option for patients with end-stage lung disease, yet reperfusion injury and resultant primary graft dysfunction continue to limit long-term lung preservation. As the need for organ replacement continues to outpace options for organ procurement, we will be investigating methods of using organs previously thought unsuitable for transplantation. Along with the use of such organs, donation from persons without beating hearts, a model of injury considered to be more severe than that seen in conventional donors, may be employed with increasing frequency. ${ }^{22}$ As such changes take place in our organ procurement protocols, we may witness an even greater rate of reperfusion injury. This potential source of morbidity and mortality further emphasizes the importance of the pathophysiology involved in this response and underscores the need for interventions designed to prevent or attenuate its deleterious effects.

In conclusion, improvement in pulmonary function after preservation and reperfusion with a neutrophil endopeptidase inhibitor confirms the role of endopeptidases in lung reperfusion injury. Further studies in large-animal transplant models are warranted to determine the potential efficacy of ulinastatin in ameliorating pulmonary ischemia-reperfusion injury and to enhance our knowledge of the detrimental effects of neutrophil endopeptidases on early pulmonary graft function.

We thank Anthony J. Herring for his technical advice, Masayuki Hotokezaka, and the Mochida Pharmaceutical Co., Ltd., Tokyo, Japan, for their gift of ulinastatin.

\section{REFERENCES}

1. Pillai R, Bando K, Schueler S, Zebley M, Reitz BA, Baumgartner WA. Leukocyte depletion results in excellent heartlung function after 12 hours of storage. Ann Thorac Surg 1990;50:211-4.

2. Schueler S, De Valeria PA, Hatanaka M, Cameron DE, Bando K, Zeebley M, et al. Successful twenty-four-hour lung preservation with donor core cooling and leukocyte depletion in an orthotopic double lung transplantation model. J Thorac Cardiovasc Surg 1992;104:73-82.

3. Weiss SJ. Tissue destruction by neutrophils. N Engl J Med 1989;320:365-76.

4. Blackbourne LH, Cope JT, Tribble RW, Tribble CG. The cardiovascular system: a brief review of the basic science of the cardiovascular system as it relates to the practice of general surgery. In: O'Leary JP, editor. The physiologic basis of surgery. 2nd edition. Baltimore: Williams and Wilkins, 1996.

5. Kennedy TP, Rao NV, Hopkins C, Pennington L, Tolley E, Hoidal JR. Role of reactive oxygen species in reperfusion injury of the rabbit lung. J Clin Invest 1989;83:1326-35.

6. Bishop MJ, Boatman ES, Ivey TD, Jordan JP, Cheney FW. 
Reperfusion of ischemic dog lung results in fever, leukopenia, and lung edema. Am Rev Respir Dis 1986;134: $752-6$.

7. DeLima NF, Binns OA, Buchanan SA, et al. Euro-Coilins solution exacerbates lung injury in the setting of high-flow reperfusion. J Thorac Cardiovasc Surg 1996;112:111-6.

8. Hall TS, Breda MA, Baumgartner WA, Borkon AM, Brown J, Hutchins GM, et al. The role of leukocyte depletion in reducing injury to the lung after hypothermic ischemia. Current Surg 1987;44:137-9.

9. Lefer AM, Lefer DJ. Pharmacology of the endothelium in ischemia-reperfusion and circulatory shock. Annu Rev Pharmacol Toxicol 1993;33:71-90.

10. Dickstein RA. Reperfusion injury: the role of leukocytes. Perfusion Life 1990; Feb:34-8.

11. Sawa Y, Matsuda H, Shimazaki Y, Kaneko M, Nishimura M, Amemiya A, et al. Evaluation of leukocyte-depleted terminal blood cardioplegic solution in patients undergoing elective and emergency coronary artery bypass grafting. J Thorac Cardiovasc Surg 1994;108:1125-31.

12. Buchanan SA, Mauney MC, DeLima NF, Binns OA, Cope JS, Shockey KS, et al. Enhanced isolated lung function after ischemia with anti-intracellular adhesion molecule antibody. J Thorac Cardiovasc Surg 1996;111:941-7.

13. Horgan MJ, Wright SD, Malik AB. Antibody against leukocyte integrin (CD18) prevents reperfusion-induced lung vascular injury. Am J Physiol 1990;259:L315-9.

14. Uthoff K, Zehr KJ, Lee PC, Low RA, Baumgartner WA, Cameron DE, et al. Neutrophil modulation results in im- proved pulmonary function after 12 and 24 hours of preservation. Ann Thorac Surg 1995;59:7-12.

15. Gorlick DL, Ortolano GA. Leukocyte depletion and implications for the prevention of reperfusion injury. Proc Am Acad Cardiovasc Perfusion 1992;13:154-65.

16. Horiguchi $T$, Harada $Y$. The effect of protease inhibitor on reperfusion injury after unilateral pulmonary ischemia. Transplantation 1993;55:254-8.

17. Endo $\mathrm{S}$, Inada $\mathrm{K}$, Yamashita $\mathrm{H}$, Takakuwa $\mathrm{T}$, Nakae $\mathrm{H}$, Yamada $\mathrm{Y}$, et al. The inhibitory actions of protease inhibitors on the production of polymorphonuclear leukocyte elastase and interleukin 8. Res Commun Chem Pathol Pharmacol 1993;82:27-34.

18. Kudo Y, Egashira T, Yamanaka Y. Protective effect of ulinastatin against liver injury caused by ischemia-reperfusion in rats. Jpn J Pharmacol 1992;60:239-45.

19. Okamoto $T$, Inagaki $Y$, Aoki $T$. Use of ulinastatin for improving viability of liver grafts. Transplant Proc 1993;25: 2961-4.

20. Li XK, Matin AF, Suzuki H, Uno T, Yamaguchi T, Harada $Y$. Effect of protease inhibitor on ischemia/reperfusion injury of the rat liver. Transplantation 1993;56:1331-6.

21. Arakawa M, Kambara K, Miyazaki H, et al. Effects of ulinastatin, an antiprotease, on alloxan-induced lung injury in dogs. Clin Ther 1992;14:396-408.

22. Buchanan SA, DeLima NF, Binns OA, Mauney MC, Cope JT, Langenburg SE, et al. Pulmonary function after nonheart-beating lung donation in a survival model. Ann Thorac Surg 1995;60:38-44. 